\title{
ATIVIDADE ANSIOLIITICA DE EXTRATO DAS FOLHAS DE MICONIA ALBICANS
}

\section{ANXIOL YTIC ACTIVITY OF EXTRACTS FROM THE LEAF OF MICONIA ALBICANS}

\author{
Kettelin Aparecida Arbos ${ }^{1}$, José Kenio Souza Nader ${ }^{2}$, Erika Nicole Luna Camelo ${ }^{3}$
}

\author{
Submetido em: 03/04/2021 \\ Aprovado em: 24/04/2021
}

\begin{abstract}
RESUMO
Miconia albicans conhecida popularmente como canela de velho é uma espécie arbórea, pertencente à família Melastomaceae, com distribuição nas regiões tropicais e com efeitos antioxidante e antiinflamatórios descritos. Embora de uso popular, não existe informações a respeito do potencial ansiolítico dessa espécie vegetal. Assim, o objetivo do trabalho foi avaliar o potencial ansiolítico de extratos hidroalcoólicos das partes aéreas de M.albicans em um modelo animal. Três concentrações diferentes do extrato bruto de $M$. albicans foram administrados intraperitonealmente e os efeitos sobre o sistema nervoso central foram analisados através do teste do labirinto em cruz elevado, teste do campo aberto e teste claro-escuro. No teste de campo aberto foi observado maior deslocamentos dos animais com o aumento das concentrações dos extratos e suspensão do comportamento de autolimpeza corporal. Animais tratados com extrato da miconia exploraram mais os braços abertos no teste em cruz elevada e tiveram maior tempo de latência no teste claro escuro. Diante destes resultados foi possível verificar efeitos ansiolíticos nos animais tratados com $\mathrm{M}$. albicans e que novos estudos devem ser realizados no sentido de identificar a substancia responsável pelos efeitos observados neste trabalho.
\end{abstract}

PALAVRAS CHAVES: Efeito ansiolítico. Ansiedade. Labirinto em cruz elevado.

\section{ABSTRACT}

Miconia albicans popularly known as "canela de velho is an arboreal species, belonging to the family Melastomaceae, with distribution in tropical regions and with described antioxidant and antiinflammatory effects. Nevertheless, despite its popular use there are no studies related to its possible anxiolytic effect of this plant species. Thus, the objective of the work was to evaluate the anxiolytic potential of hydroalcoholic extracts from the aerial parts of M.albicans in an animal model. Three different concentrations of the crude extract of $M$. albicans were administered intraperitoneally and the effects on the central nervous system were analyzed using the elevated plus maze test, open field test and light-dark box. In the open field test, greater displacement of animals was observed with increased concentrations of extracts and suspension of self-cleaning behavior. Animals treated with miconia extract explored more open arms in the elevated cross test and had longer latency times in the light-dark test. In view of these results, it was possible to verify anxiolytic effects in animals treated with M. albicans and that further studies should be carried out in order to identify the substance responsible for the effects observed in this work.

KEYWORDS: Anxiolytic effects. Anxiety. Elevated plus maze.

\section{INTRODUÇÃO}

A ansiedade é, dentre os quadros psiquiátricos, uma das patologias de maior prevalência. Estima-se que 10 a $30 \%$ da população mundial sofra de ansiedade. Diante deste cenário, muitas

\footnotetext{
1 Docente de Microbiologia do CTDR da Universidade Federal da Paraíba - UFPB.

2 Universidade Federal da Paraiba - UFPB

3 Faculdade de Medicina Nova Esperança - PB
} 


\section{RECIMA21 - REVISTA CIENTÍFICA MULTIDISCIPLINAR}

ATIVIDADE ANSIOLÍTICA DE EXTRATO DAS FOLHAS DE MICONIA ALBICANS Kettelin Aparecida Arbos, José Kenio Souza Nader, Erika Nicole Luna Camelo

pesquisas têm sido realizadas na busca de alternativas mais naturais no controle das manifestações geradas em um quadro de ansiedade (PINTO el al. 2012; SOUSA, OLIVEIRA e CALOU, 2018).

O gênero Micônia possui milhares de espécies, sendo relatadas no Nordeste Brasileiro a ocorrência de mais de 250 espécies diferentes (OLIVEIRA et al., 2014; PIERONI e DOKKEDAL, 2017). Estudos farmacológicos com extratos deste gênero são escassos, mas há descrição de atividade biológica, como antimicrobiano (CELOTTO el al, 2003); antinociceptiva (SPESSOTO et al., 2003), anti-inflamatória (VASCONCELOS et al., 2006) e antioxidante (PIERONI et al., 2011).

Até o presente momento não foram encontrados dados sobre ação da Miconia albicans sobre o sistema nervoso central (SNC), mas há consenso que muitas plantas encontradas na natureza exibem efeitos psicotrópicos e são capazes de afetar as funções do SNC exercendo importantes efeitos sobre a consciência, as emoções e a cognição (BRASIL, 2019). Pinto el al. (2012) relatam que o estudo da fisiopatogenia das doenças neuropsiquiátricas e do mecanismo de ação e da eficácia de terapias farmacológicas depende diretamente da existência de modelos experimentais (testes comportamentais e neurológicos). Neste sentido, os modelos animais e ensaios neuroquímicos podem fornecer informações úteis para o desenvolvimento de novos psicofármacos (BARETTA, 2012; CRUZ, LANDEIRA-FERNANDEZ, 2012; SILVA,2015).

Desta forma, a presente pesquisa se propôs avaliar a ação ansiolítica in vivo de extratos obtidos de folhas de Miconia albicans através de modelos comportamentais.

\section{METODOLOGIA}

\section{- MATERIAL VEGETAL E PREPARO DO EXTRATO}

As folhas de Miconia albicans foram coletadas no município de Souza -PB, nos meses de março e abril de 2017. O extrato foi preparado com as folhas secas, moídas e maceradas em etanol/água $(70: 30 \mathrm{v} / \mathrm{v}) / 7$ dias. Sequencialmente foi realizado concentração em evaporador rotativo em pressão reduzida e baixa temperatura obtendo-se o extrato bruto. Para ensaio in vivo este extrato foi diluído em solução fisiológica para obtenção das concentrações testes.

Para os tratamentos experimentais foram realizadas 3 diluições do extrato seco $(25 \mathrm{mg} / \mathrm{kg}$; $50 \mathrm{mg} / \mathrm{kg} ; 75 \mathrm{mg} / \mathrm{kg})$, tendo como controle positivo diazepam $(2 \mathrm{mg} / \mathrm{kg})$ e solução fisiológica como controle negativo. Os extratos da planta e o diazepam foram diluídos em solução fisiológica para serem administrados através da via intraperitoneal (i.p.).

- ANIMAIS

Foram empregados 50 ratos Wistar machos com peso médio de $300 \mathrm{~g}$, provenientes do Biotério João Bezerra de Lima das Faculdades Nova Esperança (FAMENE). Os animais foram 


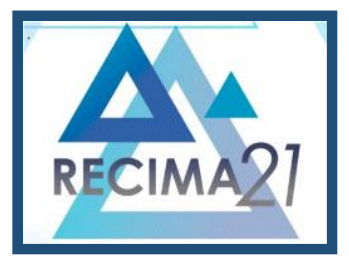

\section{RECIMA21 - REVISTA CIENTÍFICA MULTIDISCIPLINAR}

divididos em 5 grupos experimentais: Controle Positivo Diazepam (CTP); Controle Negativo (CTN); Teste Miconia 25 (M25), Teste Miconia 50 (M50) e Teste Miconia 75 (M75). Até o momento da realização dos testes comportamentais os animais foram mantidos sob um sistema claro/escuro de $12 \mathrm{~h}$, com temperatura $(23 \pm 2 \stackrel{\circ}{\circ})$ e umidade ( $55 \pm 15 \%$ ) controladas. A alimentação dos animais foi constituída por ração balanceada padronizada para roedores, e a água ofertadas ad libitum.

No momento da realização dos testes, os animais foram retirados das gaiolas e submetidos a administração dos extratos, diazepam ou solução fisiológica, de acordo com o grupo experimental no qual se encontrava, sendo na sequencia expostos aos testes do labirinto em cruz elevado, claroescuro e campo aberto. O estudo foi aprovado pelo CEAU (Certidão n. ${ }^{\circ}$ 0058.2017.1).

\section{- MODELO EXPERIMENTAL CAMPO ABERTO}

Este teste foi empregado para avaliar a atividade exploratória de cada animal após o tratamento com as substâncias de interesse. $O$ aparato foi confeccionado em madeira $(60 \times 45 \times 20 \mathrm{~cm})$ e seu interior dividido em 9 quadrantes demarcados com fita adesiva conforme o esquema representativo (Figura 1).
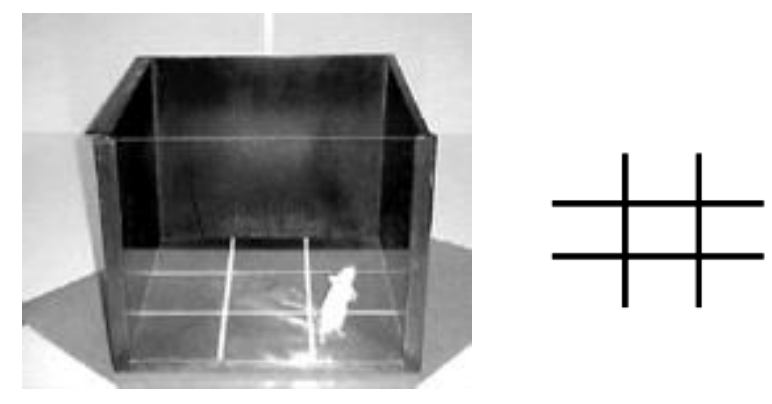

FIGURA 1 - Desenho esquemático do aparato empregado para o teste do campo aberto.

Após 30 minutos dos tratamentos experimentais, os animais, um por vez, foram colocados no centro do campo aberto onde foram registrados o número de cruzamentos com as quatro patas (atividade locomotora espontânea), número de comportamentos de autolimpeza (grooming) e o número de levantamentos (rearing), sem se encostar nas paredes, durante um tempo de 5 minutos.

\section{- MODELO EXPERIMENTAL DO LABIRINTO EM CRUZ ELEVADO (TLCE)}

O modelo do labirinto foi confeccionado em madeira com 4 braços em forma de cruz, com dois braços abertos $(50 \times 10 \times 20 \mathrm{~cm})$ e dois braços fechados $(50 \times 10 \mathrm{~cm})$, conforme esquema ilustrativo (Figura 2). Tem como princípio básico a geração de respostas comportamentais no animal 


\section{RECIMA21 - REVISTA CIENTÍFICA MULTIDISCIPLINAR}

ATIVIDADE ANSIOLÍTICA DE EXTRATO DAS FOLHAS DE MICONIA ALBICANS Kettelin Aparecida Arbos, José Kenio Souza Nader, Erika Nicole Luna Camelo

semelhantes as de humanos em situações de ansiedade, onde as drogas ansiolíticas favoreceriam o aumento do número de entradas e a permanência do animal nos braços abertos (CRUZ e LANDEIRA-FERNANDES, 2012).

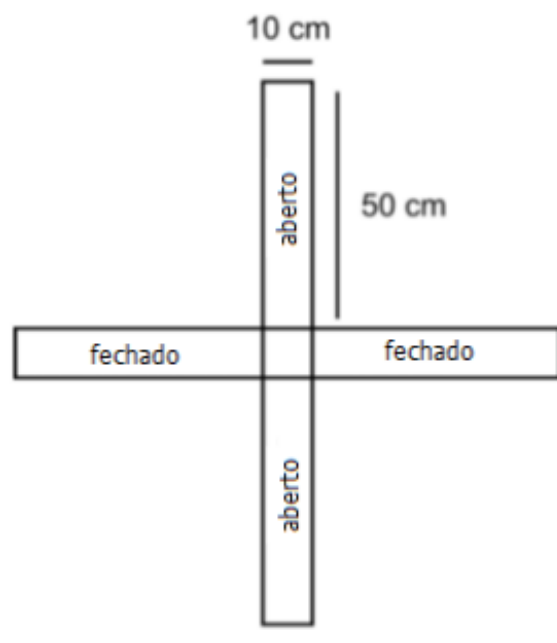

$50 \mathrm{~cm}$

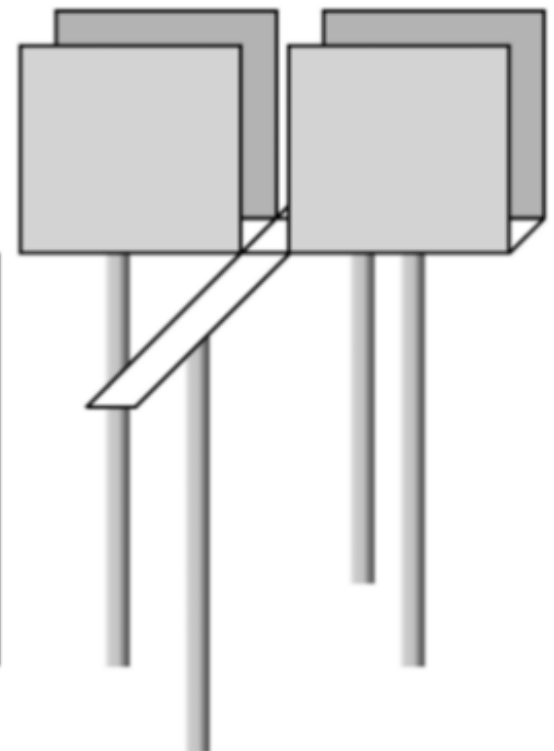

Figura 2 - Diagrama esquemático do TLCE.

Após 30 minutos da administração das substâncias (diazepam, solução fisiológica ou extratos vegetais), cada animal foi exposto ao modelo individualmente e observado seu comportamento por 5 min. Os parâmetros avaliados foram o número de entradas nos braços abertos e fechados e o tempo de permanência nos braços abertos e fechados. $O$ efeito ansiolítico foi definido como uma aumento no número de entradas nos braços abertos e o tempo gasto nos mesmos relativo ao tempo total. A entrada nos braços fechados foi utilizada como medida da atividade de 


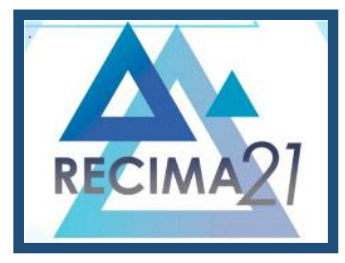

\section{RECIMA21 - REVISTA CIENTÍFICA MULTIDISCIPLINAR}

ATIVIDADE ANSIOLÍTICA DE EXTRATO DAS FOLHAS DE MICONIA ALBICANS Kettelin Aparecida Arbos, José Kenio Souza Nader, Erika Nicole Luna Camelo

locomoção dos animais (GRUNDMANN et al., 2007; DICKEL et al., 2010; PEREIRA, GUEDES e SILVA, 2015).

\section{- MODELO EXPERIMENTAL CAMPO CLARO-ESCURO}

Este teste tem como princípio básico o conflito natural que ocorre quando os animais são expostos a um ambiente não familiar, que é gerado devido à tendência natural do roedor de evitar o desconhecido. $\mathrm{O}$ aparato consiste de uma pequena caixa com dois compartimentos conectados, um branco fortemente iluminado e outro preto e escuro (MOREIRA, 2013). Ao iniciar o teste o animal é colocado no compartimento branco e pode explorar livremente os dois compartimentos (Figura 3).

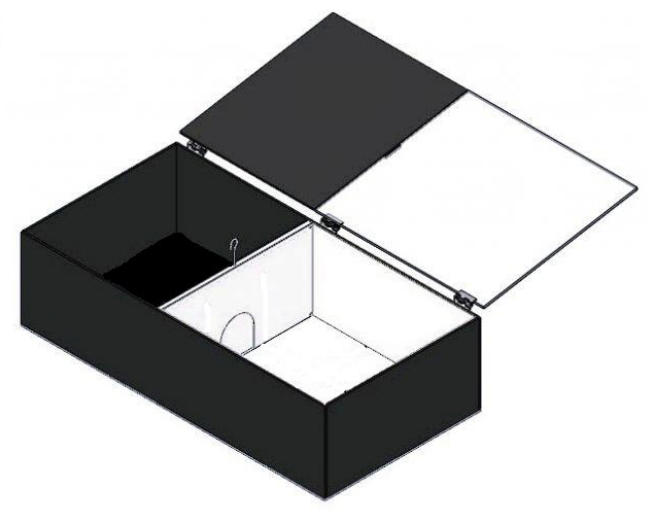

FIGURA 3 - Desenho esquematıco ao aparato empregaao para o teste do campo claro escuro

- ANÁLISE ESTATÍSTICA

Empregou-se análise de variância (ANOVA), seguido do teste de dunnett. A diferença, estatisticamente significativa, foi considerada quando o valor de $p$ for menor que 0,05 .

\section{RESULTADOS}

$\mathrm{Na}$ tabela 1 estão apresentados os resultados da ação do extrato de Miconia albicans no comportamento de ratos submetidos ao teste do campo aberto. Este ensaio permite avaliar os efeitos agudos de um extrato ou substância sobre a excitabilidade do sistema nervosa central (SNC). Para medir a atividade motora do extrato mensurou-se o número de deslocamentos espontâneos que os animais realizaram no campo aberto, os quais estão apresentados na Figura 1. Neste teste não foi verificado prejuízo na mobilidade dos animais tratados com as diferentes concentrações dos extratos da planta (M25, M50 e M75). Em relação ao número de cruzamentos houve um aumento na passagem dos animais de um campo ao outro com o aumento da concentração dos extratos, ou seja, doses maiores aumentaram a capacidade exploratória dos animais.

Segundo Barbosa e Lima (2016) o aumento no número de cruzamentos está relacionado com a exploração, o que sugere um efeito ansiolítico da substância, por outro lado, a diminuição da 


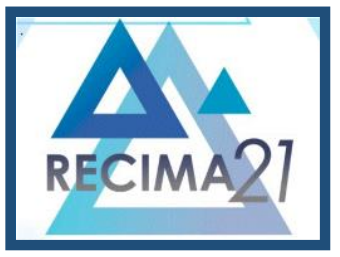

\section{RECIMA21 - REVISTA CIENTÍFICA MULTIDISCIPLINAR}

ATIVIDADE ANSIOLÍTICA DE EXTRATO DAS FOLHAS DE MICONIA ALBICANS Kettelin Aparecida Arbos, José Kenio Souza Nader, Erika Nicole Luna Camelo

atividade locomotora exploratória pode estar relacionada à sedação resultante da depressão do SNC (FRANCO et al., 2005). Desta forma os efeitos observados após a administração dos extratos de M.albicans sugere um potencial efeito ansiolítico que deve ser melhor explorado.

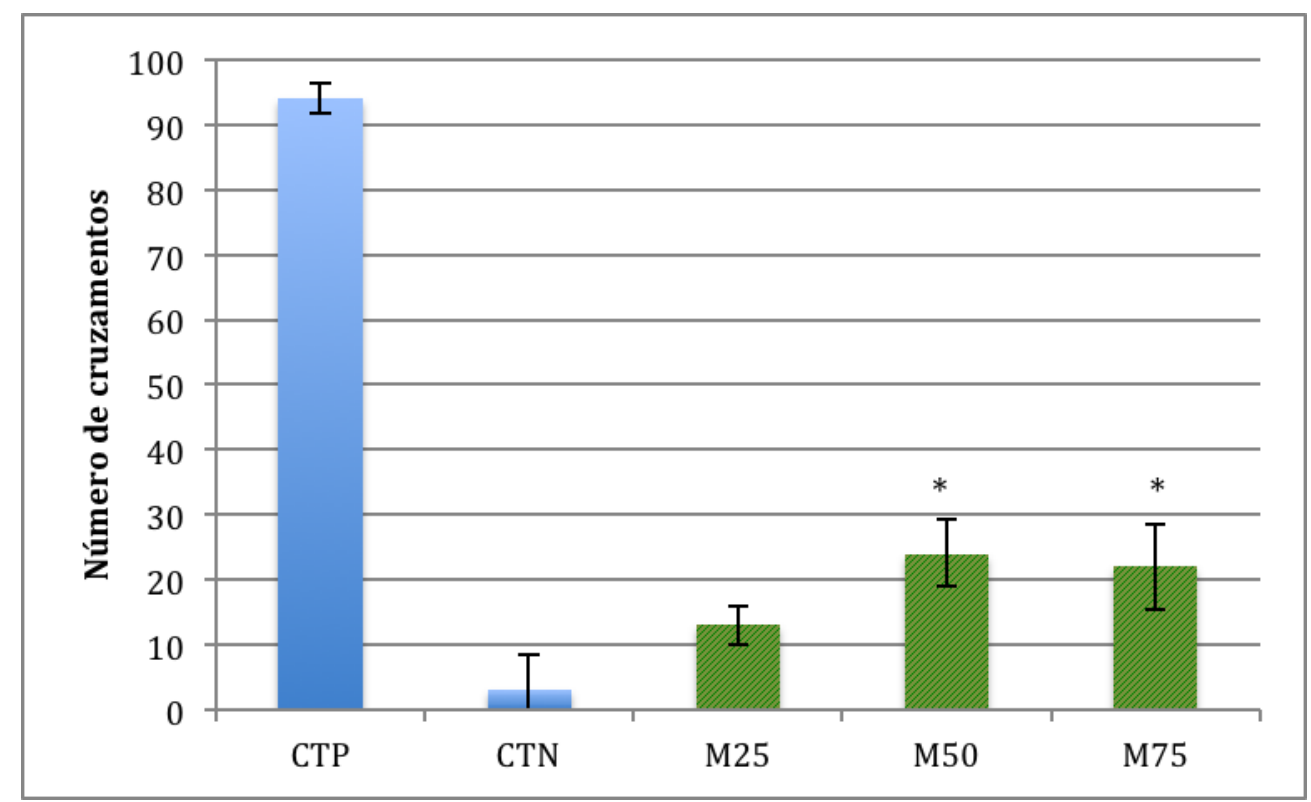

Figura 1- Efeito do extrato de M. albicans no número de cruzamentos no teste do campo aberto

CTP: controle positivo com Diazepam (2mg/kg, i.p.). CTN: controle negativo soro fisiológico $(2 \mathrm{mg} / \mathrm{kg}$, i.p.). M25: grupo tratado com extrato vegetal $(25 \mathrm{mg} / \mathrm{kg}$, i.p). M50: grupo tratado com extrato vegetal $(50 \mathrm{mg} / \mathrm{kg}$, i.p). M75: tratado com extrato vegetal $(75 \mathrm{mg} / \mathrm{kg}$, i.p). Resultados apresentados como média $\pm D P$ e analisados estatisticamente por ANOVA 1 via.

* diferença estatística significativa $(p<0,05)$ em relação aos demais grupos experimentais

\begin{tabular}{c|c|c|c}
\hline Grupos & Cruzamentos & $\begin{array}{c}\text { "Reasing" } \\
\text { (ato de levantar) }\end{array}$ & $\begin{array}{c}\text { "Grooming" } \\
\text { (ato de limpar) }\end{array}$ \\
\hline CTP & $94 \pm 2,3^{\mathrm{a}}$ & $62 \pm 7,9^{\mathrm{a}}$ & $17 \pm 3$ \\
\hline CTN & $3 \pm 3,5^{\mathrm{b}}$ & $2 \pm 2,1^{\mathrm{b}}$ & 0 \\
\hline M25 & $13 \pm 3^{\mathrm{c}}$ & $5 \pm 1,3^{\mathrm{b}}$ & 0 \\
\hline M50 & $24 \pm 5,2^{\mathrm{d}}$ & $16 \pm 1,5^{\mathrm{c}}$ & 0 \\
\hline M75 & $22 \pm 6,5^{\mathrm{d}}$ & $26 \pm 2,8^{\mathrm{c}}$ & 0 \\
\hline
\end{tabular}

Os demais parâmetros analisados foram o "reasing" que se refere ao número de vezes que o animal se levanta, ficando apenas com as patas traseiras no solo e o "grooming" que é o reflexo de autolimpeza, os quais são empregados com o intuito de avaliar possíveis modificações comportamentais.

Tabela 1 - Efeito do extrato de M.albicans sobre o comportamento de ratos no teste de campo aberto.

ISSN: 2675-6218 - RECIMA21 - Ciências Exatas e da Terra, Sociais, da Saúde, Humanas e Engenharia/Tecnologia 


\section{RECIMA21 - REVISTA CIENTÍFICA MULTIDISCIPLINAR}

ATIVIDADE ANSIOLÍTICA DE EXTRATO DAS FOLHAS DE MICONIA ALBICANS Kettelin Aparecida Arbos, José Kenio Souza Nader, Erika Nicole Luna Camelo

CTP: controle positivo com Diazepam (2mg/kg, i.p.). CTN: controle negativo soro fisiológico $(2 \mathrm{mg} / \mathrm{kg}$, i.p.). M25: grupo tratado com extrato vegetal $(25 \mathrm{mg} / \mathrm{kg}$, i.p). M50: grupo tratado com extrato vegetal $(50 \mathrm{mg} / \mathrm{kg}$, i.p). M75: tratado com extrato vegetal $(75 \mathrm{mg} / \mathrm{kg}$, i.p). Resultados apresentados como média $\pm D P$ e analisados estatisticamente por ANOVA 1 via. Grupos identificados com letras iguais não mostram diferenças significantes entre si $(p<0,05)$

Houve diferenças significativas $(p<0,05)$ ao comparar os grupos M25, M50 e M75 com o controle positivo, visto que o tratamento dos animais com diazepam ocasionou um maior número de levantamento (reasing) e autolimpeza corporal (grooming).

O comportamento de autolimpeza nos roedores pode ser exacerbado em situações estressoras. Destaca-se que os animais tratados com M.albicans suspenderam o comportamento de autolimpeza, sugerindo potencial efeito ansiolítico e antidepressor desta planta. De forma semelhante, o tratamento de animais com óleo essencial de alecrim reduziu a locomoção, reasing e grooming em relação ao grupo controle, indicando que o óleo essencial não produziu efeitos psicoestimulantes na atividade motora dos animais (ARRUDA, 2014).

Sousa et al. (2004) ao descrever a ação ansiolítica e antidepressiva de uma análogo da riparina III, extraído de riparia, demonstrou diminuição na autolimpeza. Parece, portanto, que o comportamento de limpeza no campo aberto pode ser um preditor da atividade antidepressiva, envolvendo a via dopaminérgica.

\section{Teste do Labirinto em Cruz Elevado}

O teste do labirinto em cruz elevada (TLCE) proporciona avaliação de diferentes padrões de respostas à exploração de novos ambientes. Neste trabalho avaliou-se a resposta ao medo através da porcentagem de tempo de permanência nos braços abertos e/ou fechados. A figura 2 mostra a exploração dos animais nos braços abertos, sendo que quanto maior o número de entrada, maior a ação ansiolítica da droga. Houve grande deslocamento nos braços abertos em todos os grupos analisados. Houve queda de apenas dois animais do grupo CTP tratados com diazepam. A ação observada nos grupos M50 e M75 foram significativamente superiores $(p<0,05)$ ao diazepam, droga ansiolítica clássica, demonstrando que o extrato de M.albicans apresentou, neste ensaio, importante potencial ansiolítico sem produzir relaxamento muscular e sem alterar a coordenação motora, visto que não ocorreram quedas nos grupos M.albicans. 


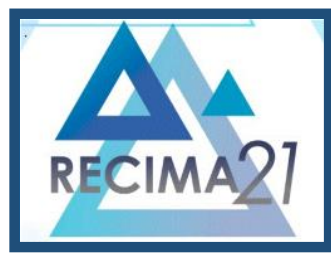

\section{RECIMA21 - REVISTA CIENTÍFICA MULTIDISCIPLINAR}

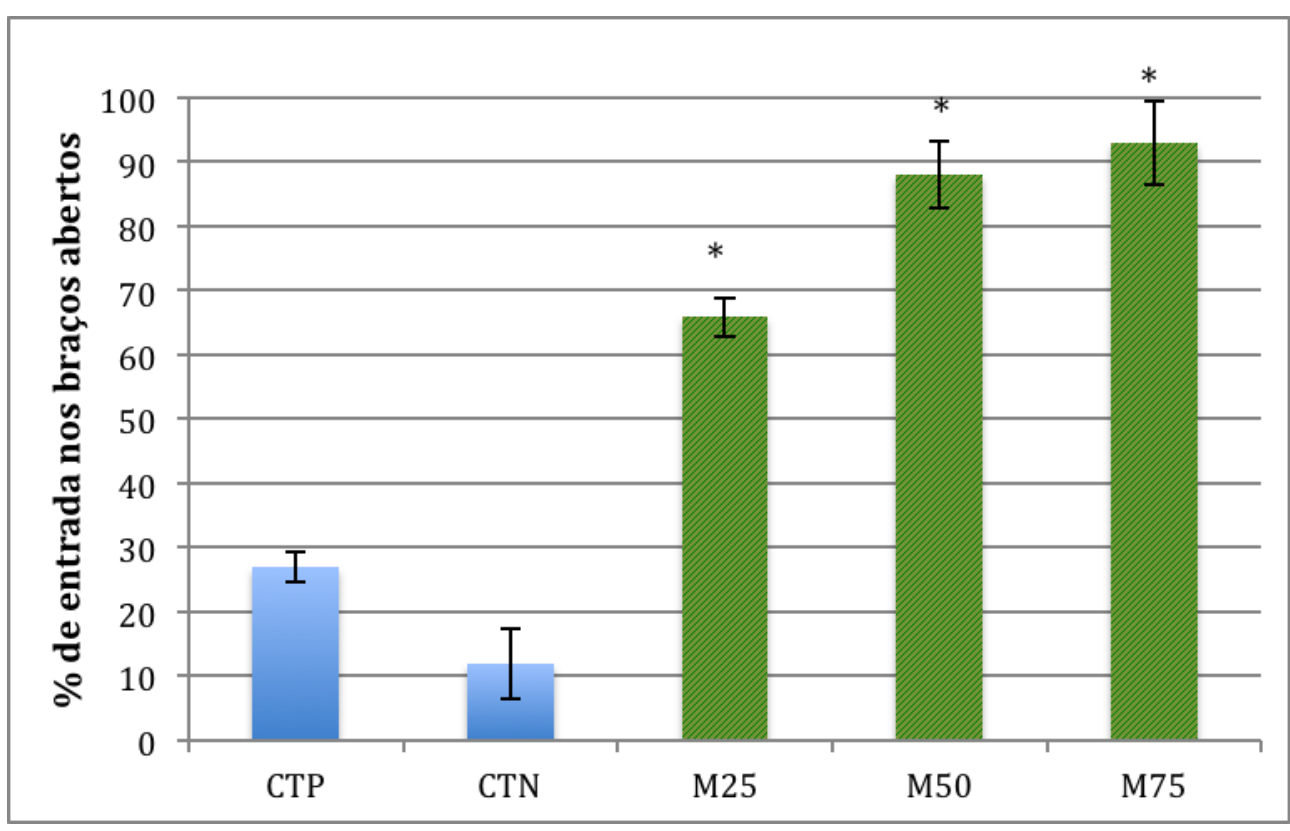

Figura 2- Porcentagem de entrada nos braços abertos do teste de labirinto em cruz elevado após tratamento extrato de M. albicans

CTP: controle positivo com Diazepam (2mg/kg, i.p.). CTN: controle negativo soro fisiológico $(2 \mathrm{mg} / \mathrm{kg}$, i.p.). M25: grupo tratado com extrato vegetal $(25 \mathrm{mg} / \mathrm{kg}$, i.p). M50: grupo tratado com extrato vegetal (50mg/kg, i.p). M75: tratado com extrato vegetal $(75 \mathrm{mg} / \mathrm{kg}$, i.p). Resultados apresentados como média $\pm D P$ e analisados estatisticamente por ANOVA 1 via.

* diferença estatística significativa $(p<0,05)$ em relação ao controle positivo $(C T P)$

Ao avaliar o tempo de permanência dos animais nos braços abertos (Figura 3), observou-se que novamente o grupo M75 diferiu do controle positivo, realizando não apenas mais cruzamentos como também permanecendo mais tempo explorando os braços abertos. Foi observada diminuição do tempo de permanência nos braços abertos tanto do grupo M50 como do M25, reforçando que a dose de $75 \mathrm{mg} / \mathrm{kg}$ foi a que apresentou melhor ação ansiolítica, visto que proporcionou uma maior frequência de entradas e mais tempo na exploração deste ambiente.

Assim como neste trabalho, Costa el al (2012) analisando o efeito de extratos de cianocarvona verificaram que doses de $75 \mathrm{mg} / \mathrm{kg}$ produziram efeitos ansiolíticos superiores ao diazepam. 


\section{RECIMA21 - REVISTA CIENTÍFICA MULTIDISCIPLINAR}

ATIVIDADE ANSIOLÍTICA DE EXTRATO DAS FOLHAS DE MICONIA ALBICANS Kettelin Aparecida Arbos, José Kenio Souza Nader, Erika Nicole Luna Camelo

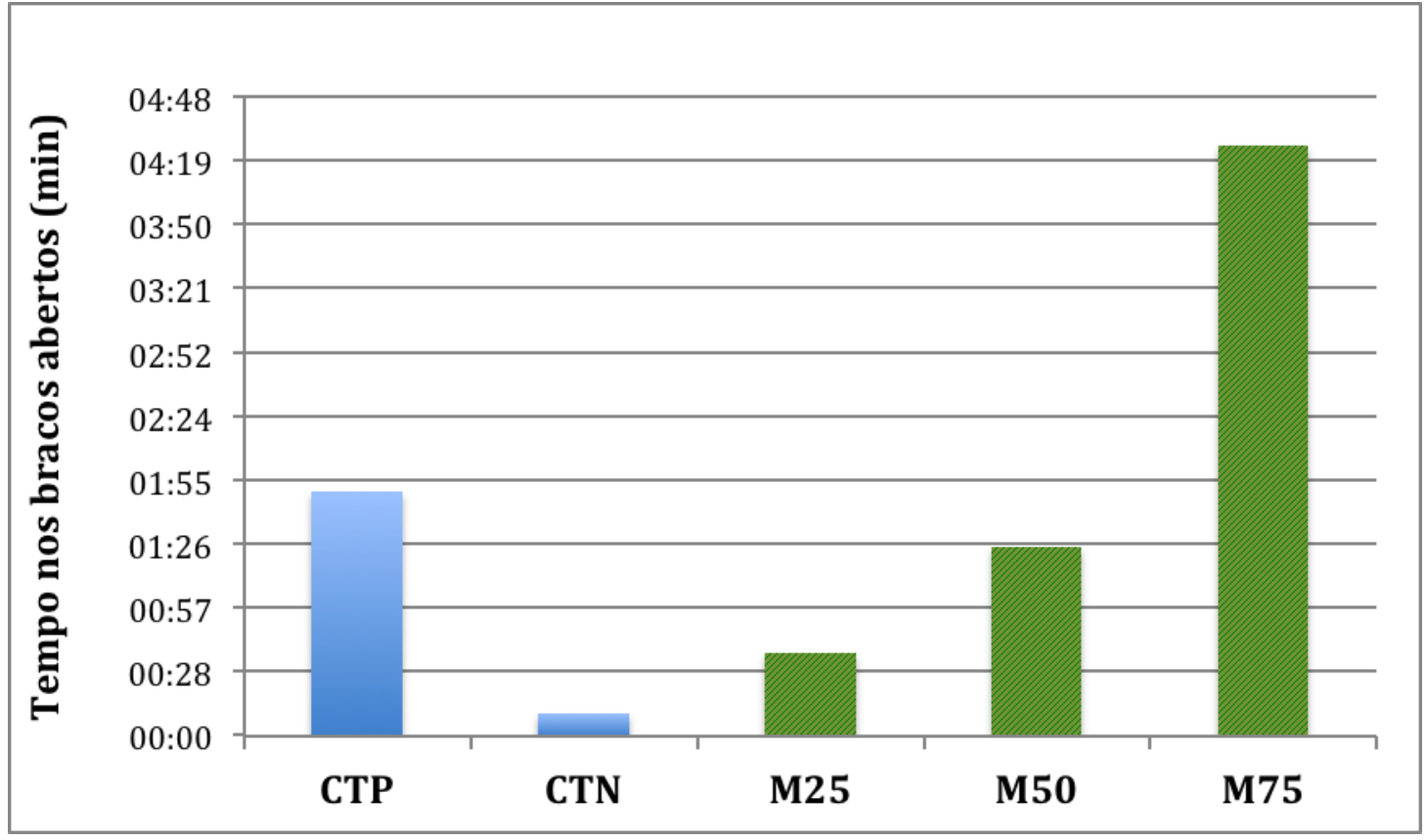

Figura 3 - Tempo de permanência nos braços abertos do TLCE

CTP: controle positivo com Diazepam (2mg/kg, i.p.). CTN: controle negativo soro fisiológico $(2 \mathrm{mg} / \mathrm{kg}$, i.p.). M25: grupo tratado com extrato vegetal $(25 \mathrm{mg} / \mathrm{kg}$, i.p). M50: grupo tratado com extrato vegetal (50mg/kg, i.p). M75: tratado com extrato vegetal ( $75 \mathrm{mg} / \mathrm{kg}$, i.p).

\section{Teste Claro e escuro}

O teste claro/escuro é comumente utilizado em neurociência comportamental para o estudo de medo e ansiedade, sendo que em geral, administrações de drogas ansiolíticas aumentam o tempo que o animal gasta no compartimento claro, enquanto drogas ansiogênicas diminuem esse tempo (MANSUR, SANTOS e GOUVEIA JR, 2014). Ao analisar o comportamento dos extratos de $M$. albicans empregando este modelo verificou-se que as concentrações de 50 e $75 \mathrm{mg} / \mathrm{kg}$ apresentaram efeitos ansiolíticos nos animais analisados (Figura 4), visto que houve aumento no tempo de latência, ou seja, o tempo que o animal demorou para acessar o lado escuro. 


\section{RECIMA21 - REVISTA CIENTÍFICA MULTIDISCIPLINAR}

ATIVIDADE ANSIOLÍTICA DE EXTRATO DAS FOLHAS DE MICONIA ALBICANS Kettelin Aparecida Arbos, José Kenio Souza Nader, Erika Nicole Luna Camelo

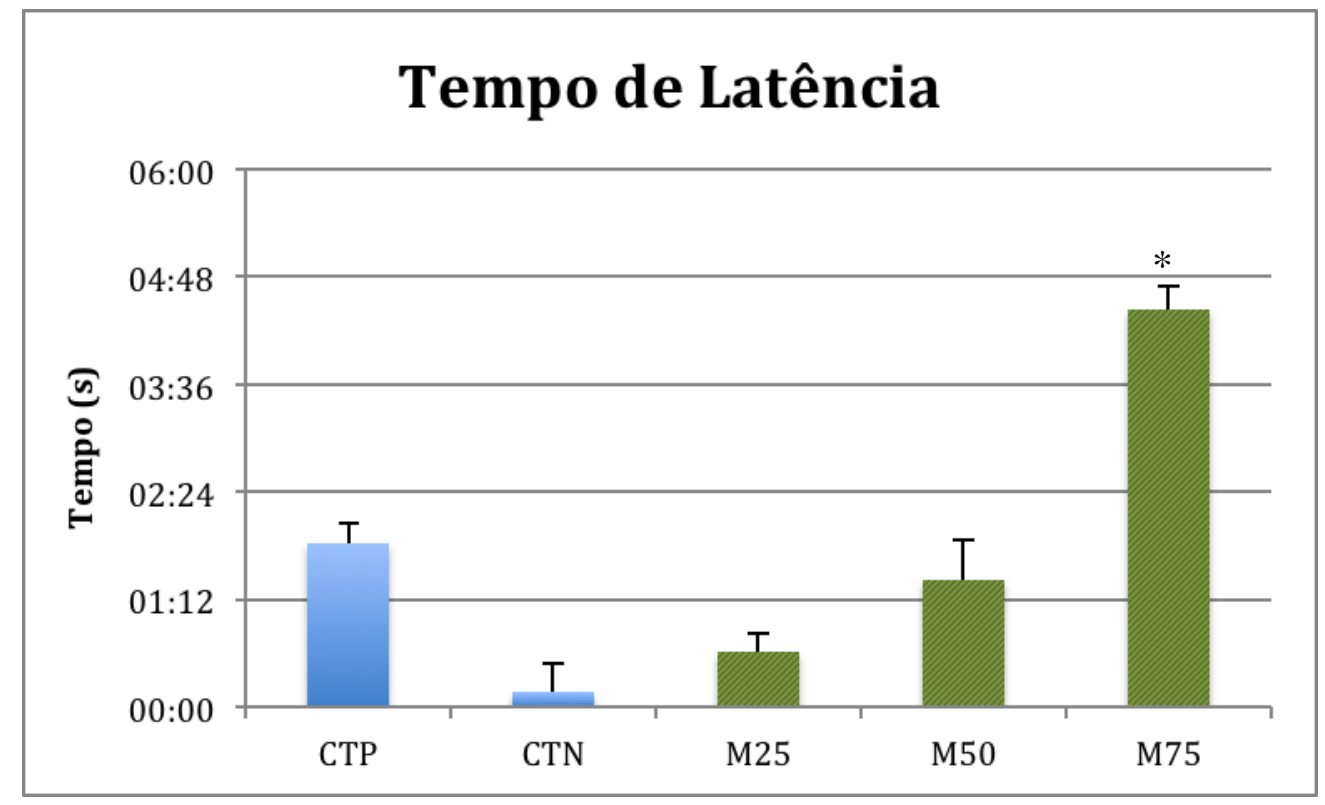

Figura 4 - Tempo de Latência no Teste de transição claro escuro.

Efeito do tratamento dos animais com extrato de Miconia albicans avaliados no teste da transição claro-escuro, registrado durante 5 minutos. CTP: controle positivo com Diazepam ( $2 \mathrm{mg} / \mathrm{kg}$, i.p.). CTN: controle negativo soro fisiológico (2mg/kg, i.p.). M25: grupo tratado com extrato vegetal $(25 \mathrm{mg} / \mathrm{kg}$, i.p). M50: grupo tratado com extrato vegetal $(50 \mathrm{mg} / \mathrm{kg}$, i.p). M75: tratado com extrato vegetal (75mg/kg, i.p). Resultados apresentados como média \pm DP e analisados estatisticamente por ANOVA 1 via.

* diferença estatística significativa $(p<0,05)$ em relação ao controle positivo (CTP).

A ação ansiolítica do extrato M50 não apresentou diferença significativa em comparação ao controle positivo ( $p \leq 0.05$ ), mas o extrato M75 apresentou ação superior ao diazepam $(p \leq 0.05)$. Neste mesmo teste são avaliados o número de transição da área clara para a escura, sendo observada $3 \pm 1$ transições no grupo M25, $5 \pm 2$ no grupo M50 e $4 \pm 1$ no grupo M75. Nos grupos controle houveram $6 \pm 2$ e $3 \pm 1$ transições para CTP e CTN, respectivamente. Desta forma, os animais tratados com os extratos de M.albicans nas concentrações de 50 e $75 \mathrm{mg} / \mathrm{kg}$ apresentaram maiores números de transições, sem diferir estatisticamente do controle positivo $(p \leq 0.05)$

\section{CONCLUSÃO}

O extrato vegetal de Miconia albicans apresentou no teste do labirinto em cruz elevado (TLCE) e no teste claro-escuro ação ansiolítica sobre SNC semelhante ao diazepam. Com a utilização do teste de campo aberto e TLCE foi possível verificar que o extrato obtido das folhas da planta analisada possui importante potencial farmacológico visto que não produziram relaxamento 


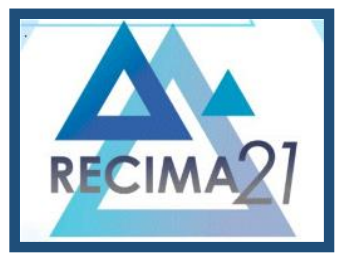

\section{RECIMA21 - REVISTA CIENTÍFICA MULTIDISCIPLINAR}

muscular e não alteraram a coordenação motora, como observado com o uso de diazepam. No entanto, são necessários mais estudos para avaliar o uso de drogas antagonistas de diferentes sistemas para esclarecer os possíveis mecanismos de ação ansiolítico, bem como identificar e isolar os principais ativos nos extratos a fim de determinar quais as substâncias que contribuem para ação clínica observada.

\section{REFERÊNCIAS}

ARRUDA, T.V. Efeitos dos óleos essências de alecrim e peitgraun em modelos comportamentais de atividade motora, depressão, ansiedade e aprendizado em ratos. 2014. 77f. Dissertação (Mestrado em Psicologia da Saúde) - Universidade Metodista de São Paulo, São Bernardo do Campo, 2014.

BARBOSA, D. F.; LIMA, C. F. Efeitos do exercício físico em comportamentos relacionados com a ansiedade em ratos. Rev. Bras. Med. Esporte, v. 22, n. 2, p. 122-125, 2016.

BARETTA, I. P. Efeito tipo ansiolítico e antidepressivo do extrato bruto hidroalcóolico de Achillea millefolium em modelos animais. 2012. 171f. Tese (Doutorado em Farmacologia) Universidade Federal do Paraná, Curitiba-PR, 2012.

BRASIL. Conselho Regional de Farmácia do Estado de São Paulo. Departamento de Apoio Técnico e Educação Permanente. Comissão Assessora de Plantas Medicinais e Fitoterápicos. Plantas Medicinais e Fitoterápicos. 4. ed. São Paulo: Conselho Regional de Farmácia do Estado de São Paulo, 2019. 72 p.

CELOTTO, A. C.; NAZARIO, D. Z.; SPESSOTO, M. A. et al. Evaluation of the in vitro antimicrobial activity of crude extracts of three Miconia species. Brazilian Journal of Microbiology, v. 34, p. 339340, dez. 2003.

COSTA, D. A.; OLIVEIRA, G. A. L.; COSTA, J. P. et al. Avaliação da Toxicidade Aguda e do Efeito Ansiolítico de um Derivado Sintético da Carvona. R. Bras. Ciencia Saúde, v. 16, n. 3, p. 302-310, 2012.

CRUZ, A. P. M.; LANDEIRA-FERNANDEZ, J. Modelos animais de ansiedade e o estudo experimental de drogas serotonérgicas. In.: LANDEIRA-FERNANDEZ, J.; FUKUSIMA, S. Métodos em Neurociência. São Paulo: Manole, 2012. p. 192-217.

DICKEL, O. E.; AGUIAR, R. B.; GERACITANO, L. et al. Efeitos comportamentais e neurotóxicos do extrato aquoso de Brugmansia suaveolens em ratos. Rev. Bras. Farm., v. 91, n. 4, p. 189-99, 2010.

FRANCO, C. I. F.; MORAIS, L. C. S. L.; QUINTANS-JUNIOR, L. J. et al. CNS pharmacological effects of the hydroalcoholic extract of Sida cordifolia L. leaves. Journal of ethnopharmacology, v. 98, p. 275-279, 2005.

GRUNDMANN, O.; NAKAJIMA, J.; SEO, S.; BUTTERWECK, V. Anti-anxiety effects of Apocynum venetum L. in the elevated plus maze test. J. of Ethnopharm., v. 110, n. 3, p. 406-411, 2007.

MANSUR, B. M.; SANTOS, B. R.; GOUVEIA JR, A. Efeitos da substância de alarme no teste claro/escuro no Zebrafish, Danio rerio. Biota Amazonia, v. 4, n. 1, p.87-93, 2014.

MOREIRA, F. A.; GOBIRA, P. H.; VIANA, T. G. et al. Modeling panic disorder in rodents. Cell Tissue Research, v. 354, n. 1, p. 119-125, 2013. 


\section{RECIMA21 - REVISTA CIENTÍFICA MULTIDISCIPLINAR}

ATIVIDADE ANSIOLÍTICA DE EXTRATO DAS FOLHAS DE MICONIA ALBICANS Kettelin Aparecida Arbos, José Kenio Souza Nader, Erika Nicole Luna Camelo

OLIVEIRA, A. K. M.; MOTA, C. M. G.; AGNES, D. C. Efeito de diferentes temperaturas na germinação de sementes e no crescimento inicial de plântulas de Miconia albicans (Melastomataceae). Rev. bras. plantas med., v. 16, n. 3, supl. 1, p. 755-759, 2014.

PEREIRA, R.; GUEDES, A. SILVA, G. E. O extrato bruto hidroalcoólico das folhas de Piper caldense C. DC. na redução do consumo de álcool em ratos. Rev. bras. plantas med., v. 17, n. 1, p. 157-163, 2015.

PIERONI, L. G.; REZENDE, F. M.; XIMENES, V. F.; DOKKEDAL, A. L. Antioxidant Activity and Total Phenols from the Methanolic Extract of Miconia albicans (Sw.) Triana Leaves. Molecules, v. 16, p. 9439-9450, nov. 2011.

PIERONI, L. G.; DOKKEDAL, A. L. Perfil cromatográfico ("Fingerprint”) do extrato metanólico das folhas de Miconia albicans (Sw.) Triana e sua atividade antioxidante. $33^{\mathrm{a}}$ Reunião Anual da Sociedade Brasileira de Química. Disponível em: http://sec.sbq.org.br/cdrom/33ra/resumos/T00111.pdf. Acesso em: 10 abr. 2017.

PINTO, W. B. V.; KO, G. M.; LAPCHIK, V. B. V.; ARIZA, C. B.; PORCIONATTO, M. Contribuições no estudo de doenças neuropsiquiátricas em modelos animais. RESBCAL, v. 1 n. 1, p. 102-120, 2012.

SILVA, J. A. C. Avaliação dos efeitos farmacológicos do óleo essencial de Chenopodium ambrosioides sobre o sistema nervoso central. 2015. 113f Dissertação (Mestrado em Ciências Farmacêuticas) - Universidade do Vale do Itajaí, Itajaí, 2015.

SOUSA, F. C. F.; MELO, C. T. V.; MONTEIRO, A. P. et al. Antianxiety and antidepressant effects of riparin III from Aniba riparia (Ness) (Lauraceae) in mice. Pharmacol Biochem Be, v. 78, p. 27-33, 2004.

SOUSA, R. F.; OLIVEIRA, Y. R.; CALOU, I. B. F. Ansiedade: aspectos gerais e tratamento com enfoque nas plantas com potencial ansiolítico. Revinter, v. 11, n. 1, p. 33-54, 2018.

SPESSOTO, M. A.; FERREIRA, D. S.; CROTTI, A. E. M.; SILVA, M. L. A.; CUNHA, W. R. Evaluation of the analgesic activity of extracts of Miconia rubiginosa (Melastomataceae). Phytomedicine, v. 10, p. 606-609, nov. 2003.

VASCONCELOS, M. A. L.; ROYO, V. A.; FERREIRA, D. A. et al. In vivo analgesic and antiinflammatory activities of ursolic acid and oleanoic acid from Miconia albicans (Melastomataceae). $\mathbf{Z}$.

Naturforsch, v. 6, p. 477-482, 2006. 